\title{
COMMENTARY
}

\section{Uncultivated microbes-in need of their own nomenclature?}

\author{
Aharon Oren and George M Garrity
}

The ISME Journal (2018) 12, 309-311; doi:10.1038/ ismej.2017.188; published online 20 October 2017

In their paper on 'Uncultivated microbes in need of their own taxonomy', Konstantinidis et al. (2017) proposed standards to draw the roadmap for a new genome-based taxonomy aimed at including the great majority of microbial species that are yet uncultivated. According to their proposal, the taxonomy of the uncultivated bacteria and archaea as based on genomic and metagenomic data, would, at least initially, be parallel but highly convergent to the one in existence for isolates. The call to include yet-to-becultivated organisms in the taxonomic system of the prokaryotes is not novel; similar proposals have been made for least two decades already. And the rapid development of sequencing technology and of bioinformatic tools to extract relevant information from the sequences obtained has already given us a wealth of information about the uncultivated majority. And there can be no doubt that the field will greatly expand in the years to come.

In the last two sections of the Perspectives paper the authors exposed their ideas about nomenclature aspects that must be taken into account when integrating the uncultivated prokaryotic world within the 'old' system that is based on isolated strains and especially type strains, representing the 15000 species of prokaryotes with names with standing in the nomenclature as regulated by the International Code of Nomenclature of Prokaryotes (the Prokaryotic Code) (Parker et al., 2016), an official publication the International Committee on Nomenclature of Prokaryotes (ICSP). Konstantinidis et al. (2017) called for the establishment of a committee of experts that should be formed to govern and supervise the new classification system, in a similar way to ICSP: 'We believe it is high time for microbial ecologists to establish their own official committee that will make recommendations on how to classify uncultured taxa with harmonized high standards, supervise and manage an 'official' classification and the rules of the nomenclature of uncultured taxa'. This is an intriguing proposal, as also for the cultivated minority such an 'official' classification system does not exist. The ICSP does not support any 'official' classification of the prokaryotes, and the Prokaryotic Code deals with nomenclature aspects only. Principle 1.4 states: 'Nothing in this Code may be construed to restrict the freedom of taxonomic thought or action'.

Konstantinidis et al. (2017) further recommended 'the implementation of an independent nomenclatural system for uncultivated taxa, following the same nomenclature rules as those for cultured bacteria and archaea but with its own list of validly published names. If widely adopted, this system will not only facilitate a comprehensive characterization of the 'uncultivated majority' but also provide a unified catalogue of validly published names, thereby avoiding synonyms and confusion'.

The Prokaryotic Code does currently not oversee the nomenclature of 'Candidatus' taxa, so that the names of 'Candidatus' taxa cannot be validly published nor have priority. The authors of the Perspectives paper therefore proposed a new nomenclature system for the uncultivated prokaryotes: 'We suggest to highlight the names of uncultivated taxa with a simple prefix such as U superscript ... which would be omitted once the organism is brought into culture ...'. Such a nomenclature system differs in nothing from that for the 'Candidatus' taxa proposed in the mid-1990s, a system that also called for omitting the 'Candidatus' when a culture becomes available for a previously uncultivated taxon. A formal proposal to incorporate the nomenclature, not only of classical 'Candidatus' taxa but also of genome and even of gene sequences to serve as type material for the valid publication of names of prokaryotes is currently pending. The 'Modest proposals to expand the type material for naming of prokaryotes' (Whitman, 2016), a document not mentioned by Konstantinidis et al. (2017), prepares the way to unite the nomenclature of the cultivated and the uncultivated prokaryotes into a single system. The proposal is complex, it includes modification of 15 Rules of the Prokaryotic Code, and it is waiting to be discussed first by the Judicial Commission and then by the plenary meeting of the ICSP in accordance with the ICSP statutes. Whether or not Whitman's 'Modest proposals' will be accepted and become part of the Prokaryotic Code cannot be predicted now. But the proposal has at least one great merit: all is done within a single framework: that of the ICSP and the Prokaryotic Code.

The idea to establish an alternative or parallel nomenclature system for the uncultivated 
prokaryotes, to be supervised 'by a committee of experts, supported by an international microbiological society (no further details provided), in order to govern and supervise the new classification system, in a similar way to ICSP' was not earlier published as far as we could ascertain. Konstantinidis et al. (2017) further recommended 'the implementation of an independent nomenclatural system for uncultivated taxa, following the same nomenclature rules as those for cultured bacteria and archaea but with its own list of validly published names. But then follows the contradictory statement that this will 'provide a unified catalogue of validly published names, thereby avoiding synonyms and confusion'.

We are greatly worried about the idea of creating independent nomenclature systems. Konstantinidis et al. (2017) proposed 'to deviate the least from the current taxonomy (intention is probably nomenclature) of cultivated taxa so that the merging of the two systems will be easy in the future'. When it is possible to remain within a single, well-established framework, that is, the ICSP and the Prokaryotic Code, what can be the advantage of first splitting and then merging? Having two independent systems running in parallel, even for a limited time, is a sure recipe for establishing synonyms and confusion. The authors stated that 'The merging would mostly depend on the implementation of two straightforward changes to the existing code of nomenclature: (i) priority of the names of uncultivated taxa is recognized by the ICNP; and (ii) DNA genome sequence is accepted as the type material ...'. These are exactly the changes proposed by Whitman (2016) while remaining within the existing formal framework.

As an example that parallel independent nomenclatural systems can work, Konstantinidis et al. (2017) quoted the case of the cyanobacteria/cyanophyta 'to which some taxonomists apply the International Code of Nomenclature for algae, fungi and plants... while the rest apply the ICNP'. This is not exactly true: most cyanobacterial taxonomists can only apply the rules of the Botanical Code as with very few exceptions only, the generic names of cyanobacteria do not have standing under the Prokaryotic Code. And for the cyanobacteria even two additional nomenclatural systems have been proposed. One is found in the paper by Gaget and coworkers cited by Konstantinidis et al. but without the comments and response published subsequently (Gaget et al., 2015). And yet another, independent, system is that of the 'Guide to the nomenclature treatment of oxyphototrophic prokaryotes' (Komárek and Golubić, 1980). Much of the current confusion was caused by a misunderstanding that in 1999 led to an unintended modification of Principle 2 of the Prokaryotic Code. The resulting pluralism, with four different nomenclature systems at the same time, has led to a tremendous confusion; for a comprehensive survey of all issues involved and the historical aspects that led to the current situation see Oren and Ventura (2017). Two opposing proposals to change the ICNP are currently pending: a proposal to largely remove cyanobacterial nomenclature from the Code (Oren and Garrity, 2014) and one to consistently apply the Rules of the Prokaryotic Code for all cyanobacteria (Pinevich, 2015). Whatever the ICSP and its Judicial Commission will decide in the future about these proposals, the single nomenclatural system that hopefully will emerge will be much easier to handle than the current pluralism.

Another example where unregulated naming of taxa is increasingly leading to chaos is that of the phyla of prokaryotes. For historical reasons the rank of phylum was never included in the taxonomic ranks covered by the Prokaryotic Code. A proposal to include the rank of phylum in the Code, co-authored by a large number of leading taxonomists (Oren et al., 2015), is waiting to be discussed by the ICSP. That proposal also includes a uniform way in which names of phyla must be formed: by the addition of the suffix -aeota to the stem of the name of one of the contained classes. A look at the many new phylumlevel named taxa proposed by Hug et al. (2016), in which not only the suffix -aeota is not used but also the regulations of Appendix 9 to the Prokaryotic Code (Parker et al., 2016) are not followed, shows how independent nomenclatural systems lead to problems that will be difficult to solve when in the future the systems must be merged to result in one coherent nomenclature acceptable to all.

For the nomenclature of the prokaryotes, cultivated as well as uncultivated, we must choose between order to be established by the ICSP (without involvement of another 'international microbiological society' in charge of the nomenclature of the uncultivated taxa) or pluralism that will inevitably lead to chaos and to the destruction of now wellordered nomenclature system.

\section{Conflict of Interest}

The authors declare no conflict of interest.

A Oren is at Department of Plant and Environmental Sciences, The Institute of Life Sciences, The Hebrew University of Jerusalem, Jerusalem, Israel G M Garrity is at Department of Microbiology \& Molecular Genetics, Biomedical Physical Sciences, Michigan State University, East Lansing, MI, USA E-mail: aharon.oren@mail.huji.ac.il

\section{References}

Gaget V, Welker M, Rippka R, Tandeau de Marsac N. (2015). Response to: 'Comments on: 'A polyphasic approach leading to the revision of the genus Planktothrix (Cyanobacteria) and its type species, $P$. agardhii, and proposal for integrating the emended valid botanical 
taxa, as well as three new species, Planktothrix paucivesiculata sp. nov. ${ }^{\text {ICNP }}$, Planktothrix tepida sp. nov. ${ }^{\text {ICNP }}$, and Planktothrix serta sp. nov. ${ }^{\text {ICNP }}$, as genus and species names with nomenclature standing under the ICNP,' by V Gaget, M Welker, R Rippka, and N Tandeau de Marsac, Syst. Appl. Microbiol. (2015). http://dx.doi.org/10.1016/ j.syapm.2015.02.004', by A Oren [Syst. Appl. Microbiol. (2015), doi:10.1016/j.syapm.2015.03.002]. Syst Appl Microbiol 38: 368-370.

Hug LA, Baker BJ, Anantharaman K, Brown CT, Probst AJ, Castelle CJ et al. (2016). A new view of the tree of life. Nat Microbiol 1: 16048.

Komárek J, Golubić S. (1980), Guide to the nomenclature treatment of oxyphototrophic prokaryotes (Cyanoprokarotes and Chloroprokaryotes). Proposal. Available at http://www.cyanodb.cz/files/CyanoGuide.pdf (accessed 24 July 2017).

Konstantinidis KT, Rosselló-Móra R, Amann R. (2017). Uncultivated microbes in need of their own taxonomy. ISME J; epub ahead of print 21 July 2017; doi:10.1038/ ismej.2017.113.

Oren A, Garrity GM. (2014). Proposal to change General Consideration 5 and Principle 2 of the International Code of Nomenclature of Prokaryotes. Int J Syst Evol Microbiol 64: 309-310.
Oren A, Ventura S. (2017). The current status of cyanobacterial nomenclature under the 'prokaryotic' and the 'botanical' code. Antonie van Leeuwenhoek 110: $1257-1269$.

Oren A, da Costa MS, Garrity GM, Rainey FA, RossellóMóra R, Schink B et al. (2015). Proposal to include the rank of phylum in the International Code of Nomenclature of Prokaryotes. Int J Syst Evol Microbiol 65: 4284-4287.

Parker CT, Tindall BJ, Garrity GM. (2016). International Code of Nomenclature of Prokaryotes. Prokaryotic Code (2008 Revision). Int J Syst Evol Microbiol; doi:10.1099/ijsem.0.000778.

Pinevich AV. (2015). Proposal to consistently apply the International Code of Nomenclature of Prokaryotes (ICNP) to names of the oxygenic photosynthetic bacteria (cyanobacteria), including those validly published under the International Code of Botanical Nomenclature (ICBN)/International Code of Nomenclature for algae, fungi and plants (ICN), and proposal to change Principle 2 of the ICNP. Int $J$ Syst Evol Microbiol 65: 1070-1074.

Whitman WB. (2016). Modest proposals to expand the type material for naming of prokaryotes. Int J Syst Evol Microbiol 66: 2108-2112. 